cp der Commissura posterior; zwischen beiden die der Commissura mollis entsprechende Durchbohrung.

Fig. 4. Palpirung des rechten Herzvorhofs vom Foramen quadrilaterum aus; schematisch. dd Zwerchfell, E Valvula Eustachii, Vorhofsseite derselben. F Fovea ovalis. co Vena coronaria cordis. tr Valvula tricuspidalis.

\title{
XXIV.
}

\section{Die neuere Literatur über menschliche und thierische Missgeburten,}

\author{
ges a m m lt von
}

Prof. Dr. E. F. Gurlt, Geh. Medicinalrath zu Berlin.

Seit der Zeit, als ich den Artikel: Monstrum in dem encyclopädischen Wörterbuch der medicinischen Wissenschaften Bd. 24. schrieb, sind viele Beobachtungen über Missgeburten gemacht worden, und ich will hier wenigstens die Literatur nachtragen, so weit sie nicht bis 1865 in Förster's Schrift: die Missbildungen des Menschen enthalten ist, die über thierische Missgeburten auch von früher.

\section{Monstra simplicia s. unicorporea.}

I. Monstra per defectum.

1. Anideus, J. G. St. Hilaire. Amorphus, Gt.

Von dieser Form sind bei dem Menschen nur zwei Fälle, der eine von Vrolik beschrieben und abgebildet, der sich von den bekannten thierischen Missbildungen darin unterscheidet, dass im Innern Wirbelrudimente mit einem Rückenmark und Nerven, und eine Darmschlinge sich vorfinden.

Eine grössere Anzahl von thierischen Missgeburten dieser Art (11 von Kühen, 1 von der Ziege) befindet sich in den Museen der biesigen und der Münchener Thierarzneiscbule. In einem Falle fand sich eine kleine Milz, Magen- und Darmanlage ä usserlich an der Missgeburt anhängend.

Literatur: W. Vrolik, Tabulae ad illustrandam embryogenesin hominis et mammalium, tam naturalem quam abnormem. Amstel. 1849. tab. 46. - 
Cornil et Eausit, Un cas de monstre Anidien chez l'homme. Gaz. méd. de Paris 1866. No. 23. - J. Glasor, Ein Amorphus globosus. Dissertat. Giessen 185̆2. - Frank, Beiträge zur Lehre von den Missgeburten. München 1875. - Gurlt, Ueber thierische Missgeburten. Berlin 1877. S. 1.

2. Acephalus, auct.

a. Acephalus trimelus. Ein Kind mit beiden oberen und der linken unteren Extremität.

Ein Ziegenlamm hat die beiden Hinterbeine und nur das rechte Vorderbein.

Lit.: Rendu, Description d'un foetus humain monstrueux acephale. Le progrès médical. No. 46. 1875. - Gurlt, Acephalus tripes. Ueber thier. Missgeb. S. 2. Taf. II. Fig. 4-6.

b. Acephalus hipes. Vier Fälle von Schafen, drei von Ziegen und einer von der Katze, ein Kind.

Lit.: Gurlt, a. a. 0. S. 1. Taf. I. Fig. 1. Taf. III. Fig. 2, 3. - Hering, Repertorium XIV. S. 97. M. Abb. - L. Calori, Annotazioni anatom. di un monstro umano acardio etc. Bologna 1870. 4. p. 36.

c. Mylacephalus. Zwei Kinder, die hierher gehören.

Li t.: Gredé, Fall von Acardiacus. Monatsschrift f. Geburtskunde. April 1869. Dieses Archiv Bd. 72. Hft. 1. 1877.

3. Pseudacephalus, Gi. Paracephalus, J. G. St. Hil.

Zwei neue Fälle sind beobachtet worden, nämlich einer rom Schaf und einer von der Ziege. Bei der Missgeburt vom Schaf enthielt die sehr kleine Schädelhöhle das verlängerte Mark und von Nerven fand sich nur der $N$. vagus und sympathicus. An der linken Seite des Halses fanden sich zwei Carotiden.

Lit.: Gurlt, a. a. 0. S. 4. Taf. II. Fig. 10-13. - Hering, im Magaz. f. d. ges. Thierheilk. XIII. S. 69. Taf. I. Fig. 1, 2.

4. Aprosopus, Gt. Triocephalus, St. Hil.

Das Fehlen des Gesichts scheint bei dem Menschen seltener vorzukommen, als bei den Thieren. Bei den Haussäugethieren, mit Ausnahme des Pferdes und der Katze, wurden 15 Fälle der Art untersucht, und einer von der Haustaube. 0 tto hat 10 Fälle beschrieben, nämlich 7 Lämmer, 2 Schweine und ein Hähnchen.

Lit.: Miram, Beschreibung zweier dem Karländischen Museum gehöriger Monstra.

Sendungen d. Kurl. Gesellsch. für Literatur und Kunst. Bd. II. No. 4. M. Abb. -

A. G. Otto, Monstrorum sexcentorum descriptio anatomica. Wratisl. 1841. p. 56-61. Tab. I. VIII. - Vrolik, a. a. 0. Tab.58. Fig. 14-16. - Gurlt, a. a. 0. S. 5 . 


\section{Microcephalus, Gt.}

Bei den Menschen ist die Microcephalie meist mit Cretinismus verbunden, und ausser der Kleinheit des Kopfes und des Gehirns sind andere Missbildungen am Kopfe nicht vorhanden. Die beobachteten Fälle dieser Art sind sehr zahlreich.

Dagegen ist bei den Thieren nicht blos der Hirnschädel zu klein, sondern er sowohl wie auch die Knochen des Gesichts sind sehr mangelhaft. Bei einem Kalbe, einem Ziegen- und einem Schaflamme wurde diese Missbildung beobachtet.

Lit.: Gurlt, a. a. 0. S. 5. Taf. III. Fig. 17-22.

\section{Agnathus et Hemignathus, Gt.}

Das Fehlen des ganzen Unterkiefers oder einer Hälfte scheint beim Menschen sehr viel seltener vorzukommen, als bei den Thieren, denn von diesen ist eine grosse Zahl von Beobachtungen bekannt. Von den drei Formen ist die erste (Mund eine Längenspalte) am häufigsten (39 Fälle) besonders bei $\mathrm{Schafen}$, während von den beiden anderen Formen nur 16 Fälle vorkamen. Aus der anatomischen Sammlung der Breslauer Universität hat 0 t to 22 Lämmer, ein Kalb und ein Schwein beschrieben.

Lit.: 0tto, a. a. 0. p.113-124. Tab. III. Fig. 2. - Vrolik, a. a. 0. Tab. 58. Fig. 11-13. Tab. 59. - Gurlt, a. a. 0. S. 6. Taf. IV. Fig. 23-25.

\section{Cyclops. Cyclopie.}

Ueber Cyclopie bei Menschen habe ich nichts nachzutragen, dagegen sind bei den Thieren viele Fälle der Art beobachtet worden, denn in der hiesigen Thierarzneischule, in deren Museum, befinden sich 74 Cyclopen und davon 26 vom Schweine. Otto beschreibt 36 Cyclopen, wovon 15 vom $\mathrm{Sch}$ weine und 14 vom S chafe sind. Bei den Formen: Cycl. perostomus und C. astomus wiederholen sich die entsprechenden Formen von Agnathus. Von verschiedenen Autoren werden auch die Fälle zu den Cyclopen gezählt, in welchen bei dem Mangel des Unterkiefers beide Augen an der unteren Fläche des Oberkiefers liegen (Agnathus astomus G.). Aber bei den ächten Cyclopen liegen die Augen immer auf der oberen Fläche des verschmolzenen Oberkiefers, auch ist bei diesen das Gehirn immer sehr mangelhaft, während es bei Agnathus astomus gewöhnlich regelmässig ist, mit Ausnahme der Kleinheit oder des Fehlens der Zungennerven. 
Lit.: J. G. Wedekind, De Cyclopia. Diss. Groningae 1830. M. Abb. H. G. Bartels, Agnus cyclops. Diss. Marburg 1840. M. Abb. $-0 \mathrm{tt} o$, a. a. 0. p. 96-110. Tab. II. Fig. 1-3. - Vrolik, a. a. 0. Tab. 55-58. Gurlt, a. a. 0. S. 29. Taf. XIV. Fig. 73-76.

8. Anophthalmus, Anommatus, Gt.

Die Augäpfel fehlen ganz, aber die Augennerven und Muskeln, sowie die Thränenorgane und Augenlider sind zugegen. Die beiden Sehnerven verbinden sich vor dem Trichter zu einem Gürtel, erreichen daher die Augenhöhlen nicht. 0tto führt nur 2 Fälle an, nämlich ein Kalb und ein Hündchen, welchen die Augen fehlen.

Lit.: v. Hasner, Sechs Fälle von Anophthalmus congenitus. Prager Vierteljahressebrift für practische Heilkunde. Bd. 20. 1876, - 0tto, a. a. 0. p.81, 82. - Gurlt, a. a. 0. S. 7. Taf. IX. Fig. 59-61.

\section{Brachyrrhynchus, Gt.}

Ein hierher gehörender Fall rom Menschen ist mir nicht bekannt, aber an Säugethieren und Vögeln habe ich mehrere Beobachtungen gemacht, nämlich bei 5 jungen Sehweinen, 3 Lämmern, einem Kalbe, einer Gans und einer Ente.

Lit.: Gurlt, a. a. 0. S. 7 .

\section{Pseud-Acormus, Gt.}

Ausser den von Förster a. a. 0. angefübrten Fällen vom Menschen, ist mir keine neuere Beobachtung bekannt geworden. Ich habe 5 solche Missgeburten von Kühen untersucht, und 0 t to und Ercolani haben auch jeder eine solche beschrieben und abgebildet.

Lit.: 0tto, a. a. 0. p. I. Tab. XXX. - Vrolik, a. a. 0. Tab.62. Fig. 4-6. Ercolani, Osservazioni teratologiche sopra un Pseudacormo bovino. Academla delle scienze dell' Istituto di Bologna. 1876. c. tav. - Gurlt, a. a. 0. S. 3. Taf. III. Fig. $7-9$.

\section{Anaedoeus, Gt.}

Das Fehlen der Geschlechtsorgane bei Menschen ist in der neueren Zeit mebrfach beobachtet worden. Die Fälle, in welchen ein Hode oder beide fehlten, hat Gruber zusammengestellt. Einem weiblichen Kinde, welches am linken Beine keinen Fuss hatte, fehlten die äusseren Geschlechtstheile. In einer Furche der Haut befand sich eine Oeffnung durch welche der Urin abging (Müller). Die Geschlechtstheile und der After fehlen, bei glejchzeitiger Sirenen- 
bildung (Banister). In einem anderen Falle fehlte auch eine Unterextremität (Hall).

An eineṁ Lamme mit Bauchspaltung fehlen alle Geschlechtsorgane, sowie die linke Niere und die Harnblase. -- Bei einem anderen Lamme, ohne After, fand sich nur die Clitoris. Bei einem Schweinchen mit Blasenspaltung fehlte die männliche Ruthe (Gurlt).

Lit.: A. E. Mäller, Verschiedene Missbildungen bei einem neugebornen Kinde. Ugeskrift for Laeger. 3. R. V. S. 329. 1869. - W. Gruber, Congenttale Anorchie beim Menschen. Oesterr. med. Jahrb. No. 1. 1868. - A. Meadows and A. J. B an is ter, Case of monstrosity. Transact. of the obstetric Soc. IX. 1868. p. 112. - A. Hall, A Case of monstrosity. ibid. p. 271. - Gurlt, a. a. 0. S. 10. S. 23. Taf. X. Fig. 63,64 .

\section{Amelus, Förster. Peromelus apus, Gt.}

Die oberen und unteren Extremitäten fehlten einem Kinde (Whitney). Von Thieren sind nur zwei Fälle zu berichten, nehmlich von einem Kalbe und einem Entchen (G.).

Lit.: 0. Whitney, Boston med. Journ. 1874. April. - Gurlt, a. a. 0. S. 11. Taf. VI. Fig. 41.

\section{Abrachius, Först. Peromelus achirus, Gt.}

Beide oberen Extremitäten fehlten einem $4 \frac{1}{2}$ jährigen Kinde (Hulke). Bei den Thieren wurden 8 neue Fälle beobachtet, nämlich 5 bei Lämmern, einer beim Pferde, einer beim Kalbe und einer beim Hasen (G.). Otto beschreibt 2 Hündchen ohne Vorderbeine.

Lit.: Hulke, Case of complete absence of both the upper limbs. The Lancet. 1872. Decbr. 2. - Otto, a. a. 0. p. 144. Tah. XIV. Fig. 2. - Gurlt, a. a. 0. S. 11. Taf. VI. Fig. $42,43$.

\section{Monobrachius, Först. Peromelus monochirus, Gt.}

Einem Kinde fehlte der linke Arm, am rechten Arme fehite der Vorderarm, aber die Hand bestand aus dem Daumen und Zeigefinger (Sedillot). Bei einem Drillingskinde, das auch sonst sehr mangelhaft gebildet war, fand sich statt des rechten Armes nur ein missgebildeter Finger (Metzner). Von den Haussäugethieren habe ich überhaupt 8 Fälle beobachtet und 0 tto beschreibt ein Hündchen, dem das rechte Vorderbein und ein Hühnchen dem der linke Flügel fehlt.

Lit.: H. Metzner, De insigni quodam partu trigemino. Diss. Halis Sax. 1851. 
- Sédillot, Sur un cas singulier de monstrosité. Comple rendu 78. No. 6 . 1874. - 0tto, a. a. 0. p. 145. - Gurlt, a. a. 0. S. 11.

15. Peromelus ascelus, Gt. Apus, Fồrst.

Von menschlichen Missgeburten, welchen beide untere Extremitäten fehlen, ist ausser den von Förster (a. a. 0.) gesammelten Beobachtungen kein neuer Fall angegeben. Bei den Haussäugethieren kam dies 4 Mal vor, namentlich bei 2 Lämmern, einem Kalbe und einem Schweinchen.

Lit.: Gurlt, a. a. 0. S. 12 .

16. Peromelus monoscelus, Gt. Monopus, Först.

Auch von dieser Art sind beim Menschen keine Fälle verzeichnet. Von Thieren sind 5 Fälle zu bericbten, wovon einer von 0 tto und vier von mir beschrieben sind. In zwei Fällen waren die Thiere vollständig erwachsen (Schwein und Ziegenbock); ein Fall betraf einen Frosch, dem das rechte Hinlerbein fehlte.

Lit.: 0 tto, a. a. 0. p.166. - Gurlt, a. a. 0. S. 12.

\section{Peromelus miccomelus, Gt.}

Ich zähle hier nur solche Fälle auf, wo einzelne Theile an den Extremitäten fehlen, und stelle solche, wo die Gliedmaassen $z$ war alle Theile enthalten aber zu klein sind, zu den Zwergbildungen.

Als neue Beobachtungen werden genannt:

Backer Brown, der ein Kind beschreibt, dem alle vier Extremitäten durch intrauterine Abschnürung im hohen Grade verstümmelt waren.

Einer 40jährigen Frau waren beide Arme mangelhaft (Hutchinson). Einem Kinde fehlte der rechte Vorderarm und linke Unterschenkel (Charon).

Bei einem anderen $\mathrm{K}$ inde endigte der linke Arm mit einem Finger (v. Ritter).

Von allen Haussängethieren ist eine beträchtliche Anzahl von Fällen (21), bei welchen entweder nur eine Extremität, oder mehrere in verschiedenen Graden defect waren, beschrieben wordell.

Lit.: Baker Brown, Obstetr. transact. VIII. p.102. 1862. - Hutchinson, Portr. of a case of congenital absence of both upper extremities. Ibid, p. 425. Schnelle, Ueber angebl. Defect von Fibula und Radius. Diss. Göttingen 1875. - Charon, Hemimelie et ectromelie. Journ. de médec. de Bruxelles, 
1876. Novbr. - v. Ritter, Ein Fall von angeborner Lücke des Brustkorbes. Oesterr. Jahrb. für Pädiatrik. 1876. Hft. 2. -0 t to, a. a. 0. p. 144. Tab. XIV. Fig. 3. p. 165. - Gurlt, a. a. 0. S. 12. Taf. VII. Fig. 44-46.

\section{Phocomelus, St. Hil.}

Diese Missbildung, welche bei Menschen sowohl bei Kindern, als auch bei Erwachsenen, nicht selten beobachtet ist, scheint bei den Thieren gar nicht vorzukommen, denn die von J. G. St. Hilaire angeführten Fälle gehören wohl nicht hierher. Neue Beobachtungen bei Menschen sind folgende; sie betreffen meist die oberen Extremitäten.

Lit.: L. Blancbmeister, Bericht über eine interessante Missgeburt (Phocomele). Zeitschrift für Medicin, Chirurgie u. Geburtshülfe. 1868. No. 4. Larrey, Phocomelie thoracique anilatérale gauche. Gaz. des hôplt. 1870. No. 92. - E. Dreibholz, Beschreibung einer sogen. Phokomele. Dissert. Berl. 1873. - Hetzell, Monstrosity poss, from material impression. Philad. med, reporter. 1876. Novbr. 25.

\section{Mononephrus, Gt.}

In mehreren Fällen war nur eing Niere vorhanden. Die rechte Niere fehlte bei eiuem Erwachsenen, die lioke war gesund aber vergrössert (Rootes). In zwei Fällen fehlte die linke Niere bei erwachsenen Personen (Bruce, Murray). Von den Thieren ist mir nur ein Fall bekannt; bei einem Schweinchen mit Blasenspaltung feblte die linke Niere.

Lit.: S. Rootes, Absence of right kidney. The Lancet II. 1866. No.9. -

A. Bruce, A solitary kidney, the bladder having but one ureter. Transaet. of the pathol. Soc. 1867. XVII. p. 175. - C. Murray, Report of a case of single kidney. British med. Journ. 1866. Aug. 11. - Gurlt, a. a. 0. S. 23. Taf. X. Fig. 64 .

II. Monstrositatis per parvitatem partium.

20. Nanus s. pygmaeus, Gt. Microsomia, Först.

Ueber Zwergbildung sind einige Beobachtungen aus neuer Zeit bekannt geworden.

Ein 5jähriges Mädchen war nur 22 Zoll gross, unfähig zu gehen und zu sprechen, körperlich sonst woblgebildet. Zwei Geschwister, nämlich ein Mädchen war 20 Jahre alt, $93 \mathrm{~cm}$. gross und 37 Pfund schwer; ein $\mathrm{Kn}$ abe, 17 Jahre alt, war $90 \mathrm{Cm}$. hoch und 39 Pfund schwer. 
Lit.: Longdon Down, Case of arrested developpement. Transact. of the patholog. Soc. 1870. XX. p. 419. - Brodowski, Aerztl. Gesellsch. Medycyna. Warschau 1874. 42.

\section{Micrommatus s. Microphthalmus, Gt.}

Die unvollkommene Entwickelung eines Auges oder beider ist bei den Thieren ziemlich häufig beobachtet worden. Ich habe $\mathbf{1 6}$ neue Fälle dieser Art untersucht, und 0 t to beschreibt ein solches Kalb. Miram hat ein solches Fohlen untersucht und beschrieben. Ich fand diesen Zustand bei 6 Fohlen, 5 Kälbern, 4 Lämmern, bei einem $\mathrm{Hündchen} \mathrm{und} \mathrm{einem} \mathrm{Kaninchen.}$

Lit.: Otto, a. a. 0. p.82. - C. E. Miram, Beselureibung einer merkwürdigen Bildungshemmung des Sehorgans etc. In v. A mmon's Monatschrift für Medicin, Angenheilkunde und Chirurgie. Bd. IIJ. Hft. 2. S. 1, m. Abb. - Gurlt, a, a. 0. S. 13. Taf. VII. Fig. 47.

\section{Micrognathus, Gt.}

Die ausserordentliche Kleinheit des Unterkiefers ist mir nur von Lämmern bekannt, bei denen ich sie sechsmal beobacbtet babe. Der Kopf des Thieres ist eben so gebildet wie in den Fällen, wo der Unterkiefer ganz fehlt und der Mund als eine Längenspalte erscheint. Der kleine Unterkiefer ist einem menschlichen Unterkiefer ähnlich, enthält die Fortsätze, aber keine Zähne.

Lit.: Gurlt, a. a. 0. S. 14. Taf. IV. Fig. 26, 27.

\section{Microphallus, Gt.}

Beí 2 Männern wurde der Penis zu kJein gefunden. Auch bei Thieren, besonders bei Hengsten, ist diese mangelhafte Bildung nicht selten.

Lit.: Zuckerland t, Ueber eine Bildungsanomalie der männlichen Geschlechtswerkzeuge. Oesterr. med. Jahrb. 1875. H. 3.

III. Monstrositates per fissuras alienas.

24. Schistocephalus, Gt.

Die Spaltungen des Hirnschädels und des Gesichts sind sehr manuichfach, und einige Formen kommen sehr oft vor, namentlich das Fehlen des Schädeldaches bei Kindern (Hemicephalie) und die Spaltungen im Gesicht, bei Menschen und Thieren.

1. Nenere Beobachtungen von Hemicephalie bei Kindern sind wenige, aber von Thieren sind einige interessante Fälle beob- 
achtet. Bei einem 7 Monate alten Kalbsfötus steckt das ganze Gesicht in dem sebr ausgedehnten Schlundkopfe und nur das vordere Ende des Unterkiefers mit der Zungenspitze ragen hervor. Der Unterkiefer und Zunge sind bis auf die Stirn hinaufgezogen, das Gehirn ist nur von einer dünnen Haut bedeckt.

Lit.: Gurlt, a. a. 0. S.17. Taf. IX. Fig, 50, 51.

2. Die partielle Schädelspaltung mit Gehirnwasserbruch. (Hydrencephalocele) ist bei Kindern und jungen Thieren oft vorgekommen. Die seltenste Form des Gehirnwasserbruchs wurde bei einem Fohlen und einem Kalbe beobachtet, bei welchen keine Schädelspaltung bestand, sondern durch das sehr grosse Foramen opticum batte der Bruch stattgefunden und statt des cyclopischen Auges war ein grosser häutiger Sack, der mit dem Amnion verwachsen, vorhanden.

Lit.: Gurlt, a. a. 0. S. 29. Taf, XIV. Fig. $75,76$.

3. Die Gesichtsspaltung, die in verschiedenen Graden vorkommt, ist bei Kindern und Thieren ziemlich häufig, doch sind, ausser den von Förster gesammelten Fällen, neuere Beobachtungen von Kindern mir nicht bekannt. Die Spaltung des ganzen Gesichts bis in den Hirnschädel (Schistocephalus bifidus) ist bei den Hausthieren mehrfach beobachtet, und ich kann, ausser den früher beschriebenen Fällen, 12 neue Fälle von Kälbern, einen vom Fohlen und einen vom Entehen anführen. Die halbe Gesichtss palte (Schistoc. semibifidus) reicht höchstens bis über die Mitte des Gesichts und ist auch bisweilen nicht in, sondern reben der Mittellinie, und ich babe nur 5 Beobachtungen gemacht.

Lit.: Gurlt, a. a. 0. S. 18, 19. Taf. VIII. Fig. 52, 53. Taf.X. Fig. 54, 55.

4. Spaltung der Wangen (Fissura buccalis congenita) ist bei den Thieren in drei verschiedenen Graden vorgekommen. Im niedersten Grade bat jede Wange in der Gegend des dritten Backenzałns eine (4 Linien grosse) Spalte, durch welche die Schleimhaut sackartig hervorgetreten ist, daher wahre Backentaschen bildet. Dies wurde bei einem auch sonst sehr missgestalteten Lamme beobachtet.

In einem höheren Grade reicht die Spalte bis an die Ohren, so dass die Paukenböhlen unten offen sind (Megalostomus). Diese Form kam bei $4 \mathrm{Lämmern}$ vor. Otto hat bei einem Lamme und einem Kalbe diese Spalting beobachtet. 
Der höchste Grad der Spaltung wurde bei einem Kalbe gefunden, bei welchem der Oberkiefer mit der oberen Hälfte der Wangen nach oben bis zur Stirn zurückgebogen, und der Unterkiefer mit der Zunge vom letzten Backenzahne an mit den unteren Wangenhälften nach unten gebogen ist (Schistocephalus retroflexus). Eine von Förster nicht angeführle Beobachtung bei einem Kinde wird hier nachgetragen.

Lit.: Gurlt, a. a. 0. S. 8. Taf. V. Fig. 31, 32. S. 21. Taf. IX. Fig. 57, 58. S. 21. Taf. II. Fig. 14-16. - otto, a. a. 0. p. 287. Tab. VIII. Fig. 1. -

G. G. F. Voigt, Annotationes criticae de evolutione cranii etc. Dissert. Regiomonti Pruss. 1841. c. tab.

5. Spaltungen der Oberlippe und

6. Spaltungen des Gaumens sind bei Menschen und Thieren vielfach beobachtet worden.

7. Fälle von Spaltung des Unterkiefers und der Zunge, oder der Zunge allein, sind bei Kindern in neuester Zeit nicht angegeben. Bei Thieren sind nur zwei neue Fälle vorgekommen, nämlich bei einem Kalbe und einem Schweinchen. Bei dem Kalbe ist die Zunge nicht blos der Länge nach, sondern an der Wurzel auch quer gespalten.

Lit.: Gurlt, a. a. 0. S. 20. Taf. XII. Fig. 56.

\section{Schistocormus, Gt.}

1. Neue Beobachtungen der Fistula colli congenita sind bei Menscben nicht gemacht; aber vom Schweine führt $\mathrm{Zündel}$ dergleichen an.

Lit.: Zündel, Die weisse Borste des Schweines. Deutsche Zeitschrift 1875. Bd. I. S. 175.

2. Ueber die theilweise Spaltung des Brustbeins (Fissura sterni) sind nur Beobachtungen an Thieren gemacht, nehmlich an $3 \mathrm{Kälbern}$ und einem Lamme. In allen Fällen lag das Herz frei zu Tage.

Lit.: Gurlt, a. a. 0. S. 21.

3. Von der Spaltung des Bauches (Fissura abdominis) liegen zahlreiche Beobachtungen von Menschen und Thieren vor. In einem Falle war die Spalte durch dünne Haut geschlossen, daher waren die Eingeweide nicht herausgetreten; das Rectum lag in der Scheide der Nabelschnur und der After fehlte. Das Pankreas, die. 
Harnorgane, das rechte 0 s innomin. und das Kreuzbein tehlten (Sheehy).

In einem zweiten Falle war nur ein 2 Zoll grosses kreisrundes Loch in der Bauchdecke, durch welches die Eingeweide herausgetreten waren; der After vorbanden ( $\mathrm{Davy}$ ).

Bei einem dritten Kinde war Spaltung des Blinddarmes und der Harnblase vorbanden und der Grimmdarm fehlte.

Bei den Haussäugethieren habe ich 46 Beobachtungen von verschiedenen Graden der Bauchspaltung gemacht, die nur zum Theil beschrieben sind; in mehreren Fällen war auch das Brustbein gespalten.

Li t.: Sheehy, Foetal malformation. The british med. Journ. 1875. Febr. 27. Davy, A singular case of ectopia of the abdominal viscera at birth. The med. Press and circular. 1875. Septbr. 8. - Dieses Archiv Bd.60. 1874. R. Hein, Beschreibung einer Missgeburt. Ibid. Bd. 58. 1873. - J. Philipeaux, Note sur un foetus monstreux à éventration complète. Gaz. méd. de Paris. 1873. No. 11. - C. Ringhoffer, Complur. deformit. infantis etc. Diss. Gryphiae 1860. c. tab. - C. D. Jung, Symbola ad docirinam de vitis circa abdomen congenitis. Diss. Bonnae 1825. c. tab. - Gurlt, a. a. 0. S. 22 . -0 tto, a. a. 0. S. $299 \mathrm{ff}$.

4. Spaltung des Beckens, der Harnblase, des Kitzlers, des Uterus und der männlichen Harnröhre.

a. In den Fällen, wo die Spaltung des Beckens allein, d. h. ohne Spaltung an anderen Theilen, besteht, ist wohl immer auch die Spaltung der Harnblase und des Kitzlers vorhanden. Neuere hierher gehörende Beobachtungen an Menschen sind gemacht von: Vogel, Blasenspaltung bei Männern und Frauen. Funk bei einem $17 j a ̈ h r i g e n$ Mädehen bei dem auch die Clitoris gespalten war. Einen ähnlichen Fall hat Pippingsköld mitgetheilt. Ich sah die Blasenspaltung bei einem männlichen Schweinchen, dem die männliche Ruthe fehlte.

Lit.: Vogel, Ueber angeborne Blasenspaltung. Leipzig 1868. - Funk, Wochenblatt der Gesellsch. Wiener Aerzte. 1870. No. 22. - Pippingsköld, Ectrophia vesicae subpubica hon en 13 ars flicka. Finska laekaresällsk förh. 1876. B. 17. p. 49. - Gurlt, a. a. 0. S. 23. Taf. X. Fig. 63, 64 .

b. Vier Fälle von Uterus et vagina duplex, und nur ein Fall von Uterus bicornis bei Menschen sind bekannt geworden.

In dem von Weiss heschriebenen Falle fehlte die Harnblase, und jeder Urether mündete in die Scheide seiner Seite. 
In einem zweiten Falle war zugleich Kloakbildung vorhanden. Turner fand einen Uterus bicornis; das eine Horn enthielt einen Fötus.

Bei den Thieren, wo der Uterus immer bicornis ist, kommen Fälle vor (Beterodidymus), wo jedes Horn einen besonderen Uterus mit Eierstock, Scheide und Vulva bildet, und wo nur zwei Nieren, aber zwei Harnblasen vorhanden sind.

Lit.: Weiss, Fall von Atresia ani und Uterus et vagina duplex. Dissert. Marburg 1867. - G. L. Heppn r, Einige wichtige Hemmungsbildungen der weiblichen Genitalien. Petersb. med. Zeitschrift. 1870. No. 3. - J. Aikmann, Case of a double uterus and vagina. Glasgow med. Journ. 1872. May. Gehrung, Case of a douhle uterus and vagina. Americ. Journ. Med. Soc. 1874. Octbr. - W. Turner, Malformation of the organs of generation. Edinb. med. Journ. 1866. May. p. 971. - Gurlt, a. a. 0. S. 49. Taf. XVII. Fig. 101.

c. Epispadie und Hypospadie. Nur ein Fall von Epispadie bei einem $3-4$ Wochen alten Kinde ist aus der neueren Literatur anzuführen. Bei den Thieren ist die Hypospadie nicht selten, ich habe 14 Fälle dieser Art untersucht, während von Epispadie mir kein Fall bekannt ist; am häufigsten fand ich jene bei Schafböcken. Bei einem Hengste reichte die Harnröhrenspalte von der Mitte der Ruthe bis durch die Eichel.

Lit.: Leboucg, Exstrophie complète de la vessie urinaire. Annal. de la Soc. de médec. de Gand. 1876. Mai 26. - Gurlt, a. a. 0. S. 34. Taf. XV. Fig. 84, 85 .

5. Die Spaltung am Rücken besteht entweder in dem Fehlen der Wirbelbogen, oder in der Nichtvereinigung der beiden seitlichen Hälften derselben. Fälle der letzten Art sind bei Menschen und Thieren mehrfach beobachtet worden.

Bei einem Kalbe und zwei Lämmern fand ich, bei theilweiser Spaltung einiger Wirbel in zwei seitliche Îălften und dem Fehlen der meisten oder aller Rippen einer Seite, die auf dem Rücken der Thiere liegenden Eingeweide gespalten, namentlich das hintere Ende des Schlundes und die Magen, wozu noch andere Missbildungen kamen.

Lit.: Gurlt, a. a. 0. S. 23. Taf. XI, XII. Fig. 65-67.

26. Schistosomus, Gt.

Spaltungen am Kopfe und in der vorderen (unteren) Mittellinie des Rumpfes sind bei Kindern und Thieren mehrfach beobachtet 
worden, doch sind neue Beobachtungen von Menschen nicht anzuführen. Dagegen ist die Leibspaltung mit völliger Zurückwendung der Brust- und Bauchwände (Schistosomus reflexus) bei Kälbern sehr oft vorgekommen. In einem dieser Fälle fehlen die fünf hinteren Halswirbel (3.-7.), so dass der Kopf mit den beiden ersten Halswirbeln nur durch die Haut mit dem Rumpfe verbunden war. Das Rückenmark ging am zweiten Halswirbel in eine Spitze aus, fing aber am ersten Rückenwirbel wieder an und reichte bis in's Kreuzbein. Bei einem anderen Kalbe war der Schlund vom Magen, und die vier sehr rudimentären Magen unter sich und vom Duodenum abgeschnürt, so dass dieses geschlossen anfing; die übrigen Eingeweide, mit Ausnahme der sehr kleinen Milz, waren normal.

Lit.: Gurlt, a. a. 0. S. 25. Taf, X. Fig. 68-70. Taf. XII. Fig. 71.

27. Schistomelus, Gt.

Neue Beobachtungen bei Nenschen sind von dieser Form der Spaltungsbildungen, der sogen. Krebsscheerenbildung, nicht bekannt geworden. Bei Hunden ist die Spaltung eines Vorderbeines bis zum Carpus zweimal vorgekommen.

Lit.: 0 tto, a. a. 0. p. 145 . - Gurlt, a. a. 0 . S. 26.

IV. Monstrositates per atresiam et symphysin.

28. Atretocormus. Gt.

Angeborne Verschliessungen der hohlen Organe am Rumpfe sind bei Menschen auch in der jüngsten Zeit mehrfach beobachtet worden.

1. Schlundkopf und Schlund. Eine betreffende Beobachtung bat Perier mitgetheilt, wo das obere Ende des Schlundes obliterirt war, und der Schlund mit der Luftröhre in offener Verbindung stand.

2. Darmkanal. Die Atresie der oberen Flexur des Duodenum, welche einen soliden Strang darstellte, hat Middleton beobachtet. Zwischen dem Jejunum und Ileum feblte ein Stück des Darmes, und der Darm war oben und unten geschlossen (Küttner). In einem anderen Falle war neben dem Verschluss des Dünndarmes auch Atresia ani vorhanden (Ahlfeld). Die Verschliessung des Dünndarmes an mebreren Stellen hat Wuenschel beobachtet. Auch B endz berichtet von einer Dünndarmverschliessung. 
Bei obliterirtem Grimmdarme fehlte das S romanum, die Afteröffnung war vorhanden (Charon et St. Moulin).

Die Atresie des Darmes bei Thieren habe ich nur zweimal beobachtet, nebmlich am Dünndarm der Ziege, und am Grimmdarm des Kalbes. Die Atresie des Mastdarmes bei fehlendem After, als alleinige Missbildnng, oder zugleich mit anderen Missbildungen ist nicbt selten vorgekommen. Ebenso ist die Atresia ani bei bestehender Kloakbildung sowohl bei neugebornen als erwachsenen Thieren, besonders bei weiblichen, nicht selten beobachtet worden.

Lit.: Perier, Anomaliè de l'oesophage, obliteration du bout super. Gaz. des hôpitanx. 1874. No.12. - M. Middleton, Cases of malformation. Americ. Journ. of med. Sc. 1868. Jan. p. 69. - Kü ttn er, Fall von Dünndarmatresie. Dieses Archiv 1871. Bd. 54. S. 34. - Ahlfeld, Zur Aetiologie der Darmdefecte und der Atresia ani. Archiv f. Gynäkologie. V. 1873. Hft. 2. Wuensche, Ein Fall von angebornem Verschluss des Pylorus etc. Jahrbücher für Kinderheilkunde. 1875. VIII. H. 3. - Viggo Bendz, Ugeskrift for laeger. 1867. III. No. 10. - Charon et $\mathrm{St}$. Moulin, Absence complète de rectum. Presse méd. Belg. 1874. No. 12. - Sharp, Occlusion of the rectum. New-York med. gaz. 1871. Apr. 15. - Dolgson, Case of imperfor. anus. Brit. med. Journ. 1868. Decbr, 26. - Lissauer, Atresia ani, mit Bildung eines Canalis recto-resicalis. Monatsschr. f. Geburtsk. 1868. Novbr. p. 351. Gurlt u. Hertwig, Magazin f. d. gesammte Thierheilkunde. 17. Jahrg. S.449. No. 169. - Ebend. Jahrg. 22. S. 314. No. 67. - Ebend, 4. Jahrg. S. 192. No. 209.

3. Der Verschluss der Harnröhre wurde von Duncan bei einem männlichen Kinde beobachtet. Bei einem anderen Kinde (Knabe) wurde der Abfluss des Urins durch zwei Schleimhautfalten in der Harnröhre vor dem Colliculus seminalis gehindert (Tolmatschew).

4. Atresia vaginae wurde vón Schell bei einer 30 jährigen Frau beobachtet. Männel berichtet über einen Fall von Atresia vaginae et uteri.

Die Atresie der äusseren weiblichen Geschlechtstheile habe ich bei einem Fohlen und einem Schweinchen gefunden.

Lit.: M. Duncan, Edinb. med. Journ. 1870. Aug. p. 163. - Tolmatschew, Fall von semilunaren Klappen in der Harnröhre. Dieses Archiv Bd. 49. S. 348. - A. S chell, A case of atresia vaginae. Philad. med. Times. 1875. Febr. 20.

- Männel, Atresia vaginae et uteri. Wiener allgem. med. Zeit. 1867. No. 46. p. 388. - Gurlt, a. a. 0. S. 27. Taf. XIII. Fig. 72. 
29. Sirenomeles, St. Hil. Sympus, Först.

Nevere Beobachtungen über Sirenenbildung sind folgende:

A. Hempel, Ueber Sirenenbildung. Dissert. Berlin, 1869. J. B. S. Jackson, Case of monstrosity. Bost. med. and surgic. Journ. 1869. Febr. 18. - G. Julliard, Sur un monstre symelien. Gaz. méd. de Paris 1869. No. 15. 16.

Bei Thieren ist bisher. keine derartige Beobachtung gemacht worden, ausser bei Thieren mit überzähligen Extremităten, wo die einander nahe liegenden mehr oder minder verschmolzen sind.

30. Syndactylus, Först. Aschistodactylus, Gt.

Die Verwachsung (oder richtiger die Nichtspaltung) der Finger und Zehen bei Menschen kommt zwar ziemlich häufig vor, doch sind neuere Beobachtungen nicht verzeichnet.

Die Nichtspaltung der Zehen bei Kälbern (bei Schafen und Ziegen sind Beobachtungen nicht gemacht) und der beiden mittleren Zehen bei Schweinen ist sehr oft, besonders bei Schweinen, gefunden worden.

Conf. Gurlt, a. a. 0. S. 28.

V. Situs et forma partium abnormis.

31. Situs viscerum inversus. Transpositio viscerum.

Die seitlich umgekehrte Lage der Brust- und Baucheingeweide, obne Spaltung der Wände dieser Höhlen, ist bei Menschen nicht selten, während an Thieren, ausser bei Doppelmissgeburten, keine Beobachtungen gemacht sind.

Lit.: Heckmann, Transposition of the abdominal and thoracic viscera. Americ. Journ. of med. Scienc. 1868. Jan. p. 280. - Scheele, Zwei Fälle von vollständ. Situs viscerum inversus. Berliner klinische Wochenschrift. 1875 . No. 29, 30. - Müller, Beobachtung eines Falles von Situs viscerum inversus. Zeitschr. f. pract. Medic. 1876. No.27. - Guttmann, Demonstr. eines Falles von Situs viscernm inversus, Berlin, klin. Wochenschr. 1876. No. 11. Frits che, Ein Fall von Situs viscerum perversus. Ebend. No. 19. - Bargl, Zur Casuistik des Situs viscerum mutatus. Aerztl. Intelligenzblatt. No. 24, 25. Moxon, Transposition of the thoracic and abdominal viscera. The lancet 1876. No. 25. - F. Orsi, Caso di transposizione totale dei visceri torac. e abdominale. Gaz. med. ital. Lombardo 1876. No. 5.

Die abnorme Lage einzelner Organe ist bei Menschen beobachtet; so fand Sands die Hod en bei einem 17 jäbrigen Menschen 
am Damme liegend, und Frua berichtet einen Fall von Prolapsus vesicae bei einem 6 Monate alten Mädchen.

Lit.: Sands, Abnormal position of the testicles. New-York medic. record. 1867. No. 37. p. 303. - Frua, Un caso di rovesciamento della vescica orinaria. Annali universal di Medicina. 1874. Novbr.

\section{Campylorrhinus, Gt.}

Die Krümmung des Oberkiefers und der Nase bei Säugethieren und des Oberschnabels bei Vögeln nach der linken oder rechten Seite kommt gar nicht selten vor. Ich habe 14 Fälle bei Fohlen, 3 bei Gänsen, 1 bei der Ente und 2 bei Hühnern beobachtet. Gewöhnlich ist der übrige Körper regelmässig gebildet.

Lit.: Gurlt, a. a. 0. S. 30 .

\section{Monstrositates per excessum.}

33. Dignathus et Trignathus, Gt.

Von dieser Form der Missbildungen, bei welcher unter einem Ohre, oder unter beiden, ein rudimentärer Unterkiefer mit einem, mit zwei oder drei Schneidezähnen, einer kleinen Mundöffnung und einer kleinen Zunge, vorhanden ist, kenne ich keine Beobachtung bei Menschen. Aber bei Thieren, besonders bei Schafen, ist dieser Zustand nicht selten. Mir sind 12 derartige Fälle bekannt. Lit.: 0 t to, a. a. 0. p. 247 ff. Tab. XXIII. Fig. 1, 2. - Gurlt, a. a. 0. S. 38, 39. Taf. XV. Fig. $86-88$.

\section{Notomeles, St. Hil. Opistomelophorus, Gt.}

Neuere Beobachtungen von überzähligen Extremitäten am Rücken oder Kreuzbein sind bei Menschen nicht gemacht. Auch bei den Säugethieren sind diese Fälle nicht häufig; ich habe sie nur bei einer $\mathrm{Kuh}$ und einem $\mathrm{Kalbe}$ beobachtet. $\mathrm{v}$. Baer beschreibt eine Kuh, die an der linken Seite des Halses zwei überzählige Vorderbeine hat, ein ähnliches Kalb ist von Richter beschrieben. Von Vögeln habe ich sechs Fälle untersucht, nänlich 1 Gänschen, 4 Hühner und 1 Taube; bei jedem dieser Vögel waren zwei überzählige Beine am Kreuzbein vorhanden.

Lit.: K. E. von Ba er, Ueber doppelleibige Missgeburten. S. 70. Taf. II, III, V. Fig. 4. - H. A. G. Richter, Opisthomelophorus tetrachirus. Dissert. Rostochỉ 1846. - Gurlt, a. a. 0. S. 50, 51. 
35. Gastromeles. Pygomeles, St. Hil. Emprosthomelophorus, Gt. Ancelet beschreibt ein $\mathrm{Kind}$, welches $5 \mathrm{Cm}$. unter dem Nabel zwei rudimentäre Beine batte. Auch von dieser Form der Missbildung sind Beobachtungen an Thieren als neu anzufübren.

Ein überzäbliges Hinterbein fand ich an der unteren Fläche des Beckens bei einem $\mathrm{Kalbe}$, welches doppelte äussere weibliche Geschlechtstheile hatte.

Bei 5 Hühnchen und 2 Entchen wurden 2 überzählige Beine und bei einem jungen Frosch zwei überzählige Hinterbeine unten am Becken gefunden.

Lit.: Ancelet, Note sur un cas de Pygomelie. Gaz. des hopitaux. 1869. No. 147,149 . - Gurlt, a. a. 0. S. 51. - 0tto, a. a. 0. p. $253,254$.

36. Melomeles, St. Hil. Pleuromelophorus, Gt.

Von dieser Art von Missbildung, die bei Menschen überhaupt sehr selten vorkommt, ist kein neuer Fall anzuführen.

Bei Kälbern und Lämmern ( 8 Fälle) kam ein drittes Vorderbein an der linken oder rechten Seite vor. Otto fand bei einem Froseh zwei Vorderbeine an der rechten Seite.

Bei einem Lamme wurden an der rechten Seite drei Vorderbeine beobachtet.

Ein überzähliges Hinterbein an der linken Seite sah 0 t to bei einem La m me.

Ich fand bei 2 Hühnchen und einem Gänschen drei Beine an einer Seite.

In einem, nur einmal vorgekommenen Falle bei einem Lamme waren drei rechte Vorderbeine und ein drittes (verkümmertes) Hinterbein, welches unten am Becken lag, vorhanden; also ein Fall von seitlicher und unterer (vorderer) Vermehrung der Extremitäten.

Lit.: 0 tto, a. a. 0. p. 276. Tab. XXVII. Fig. 1. Ibid. p. 256. Tab. XXVII.

Fig, 3-5. - Gurlt, a. a. 0. S. 52,53 .

37. Polydactylus, Fơrst. Perissodactylus, Gt.

Bei Menschen sind mehrere Fälle von überzähligen Fingern und Zehen vorgekommen.

Auch bei den Thieren sind zahlreiche neue Fälle beobachtet; bei Fohlen 14 Fälle, 7 bei Kälbern, 9 bei Schweinen, 3 bei Hunden, einer bei der $K a t z e$ und 2 bei der Henne. Bei den 
Einhufern ist im Normalzustande schon die Anlage $z u$ drei Zehen vorhanden, und das Vorkommen von drei und mehr Zehen ist ja bei fossilenden Pferden (Hipparion) beobachtet. In der Mehrzahl der Fälle kommen die überzähligen Zehen an den Vorderbeinen vor.

Lit.: Gurlt, a. a. 0. S. 31 ff. Taf. XIII. Fig. $77-81$. -0 tto, a. a. 0. S. $273-274$.

\section{Hermaphroditus, Gt.}

Neuere Beobachtungen von Zwitterbildung sind von Menschen nicht zu berichten.

Von Thieren sind mir drei Fälle bei jungen $\mathrm{Sch}$ weinen vorgekommen:

1. Hermaphroditus lateralis, an der linken Seite des Uterus eine Tuba und ein kleiner Eierstock, am rechten Horn ein Hode mit Nebenhode und Samenleiter, bei übrigens vollständigen weiblichen Geschlechtstheilen.

2. Hermaphroditus transversus. Bei zwei jungen weiblichen Schweinen fand ich an jedem blind endigenden Uterushorn einen Hoden mit Nebenhoden und Samenleiter.

Lit.: Gurlt, a. a. 0. S. 33. Taf. XIV. Fig. 82, 83.

39. Androgynus, Gt.

1. Androgynus masculinus. Nur von Thieren sind Beobachtungen nachzutragen, nämlich von 2 Ziegenböcken und einem Rinde. Bei dem letzten ist die seitliche mit der doppelgeschlechtigen Zwitterbildung vorhanden. Es ist nämlich am rechten Horn ein Eierstock und Tuba vorhanden, am linken ein kleiner Hode mit geschlossenem Samenleiter. Die übrigen Geschlechtsorgane sind vollständig männliche.

2. Androgynus femininus kam bei einer Ziege und einem Schafe vor. An den Enden beider Hörner des Uterus befinden sich zwei Hoden, sonst sind alle Theile weibliche.

Lit.: Gurlt, a. a. 0. S. 34, 35 .

\section{Monstra duplicia.}

I. Ohne Trennung an beiden Enden des Körpes.

40. Monocranus, Gt.

Diese Art von Missbildung, welche bei dem Menschen nicht beobachtet ist, wurde bei drei Kälbern und einem Lamme neu 
untersucht. Eine von diesen abweichende Form kam bei einem Fohlen vor, bei welchem der parasitische Oberkiefer cyclopisch war.

Lit.: Doenitz, Beschreibung und Erläuterung von Doppelmissgeburten. Reichert's u. du Bois-Reymond's archiv 1865. Taf. XII, XIII. - Gurlt, a. a. 0. S. 39. Taf. XVI. Fig. 89-92.

\section{Diprosopus, Gt.}

Von den drei bekannten Formen: Diprosopus sejunctus, conjunctus und distans ist nur die zweite, bei einem $\mathrm{Kinde}$ von Macdonald beobachtete, als neu anzuführen. Dagegen sind bei den Thieren alle drei Formen mehrfach untersucht und beschrieben. In einem Falle fand man unter dem linken Ohre des rechten Kopfes eines Lammes einen parasitischen kleinen Unterkiefer. (Vergl. oben Dignathus et Trignathus.)

Lit.: Macdonald, Description of a diprosopus triophthalmus monster, Edinb. med. Journ. 1875 . Febr. -0 t to, a. a. 0. S. 226 ff. S. 234, 235. Tab. XXIV. Fig. 4. - Gurlt, a. a. 0. S. 36, 37.

\section{Missgeburten mit Trennung am oberen (vorderen) Körperende.}

\section{Dicephalus, Gt.}

Die fünf Formen, je nach dem Grade der Verdoppelung der Wirbelsäule, sind bei Menschen und Thieren ziemlich häufig beobachtet worden. Von Kindern sind vier neue Beobachtungen mitgetheilt; nämlich von Hällström, Kohler, Werner und Berjoan. Bei den Thieren ist eine grössere Zahl von Beobachtungen gemacht worden.

Lit.: Hällström, Dicephalus tribrachius tripus. Finsk. laek. sällsk. handl. 1871. Bd. 12. S. 265. - A. Kohler, A bicipital monster. New-York med. Record. 1871. Novbr. 1. - Werner, Eine Doppelmissgeburt. Berl. klinische Wochenschr. 1876. No.32. - Berjoas, Un cas de monstre donble autositaire. Gaz. hebdom. de médec. et de chirurg. 1876. No.16. - M. A. Thi er nesse, Description d'un monstre double monomphalien. Bulletin de l'academie roy. de médec. de Belgique. T.X. No.4. - 0 tt a, a. a. 0. p. 228 fi. Gurlt, a. a. 0. S. 41 fi. - Serafinski, Sitzungsber. der Lubliner ärztl. Gesellsch. Medycyna. 1876. No. 32.

43. Thoraco-Gastrodidymus, Gt. Xiphodymus, St. Hil.

Nur eine bei dem Menschen von pasquet-Labrone gemachte Beobachtung aus der neueren Zeit ist zu berichten. Bei 
den Thieren ist diese Form nur zweimal vorgekommen, nämlich bei einem $\mathrm{Ziegenlamme} \mathrm{und} \mathrm{einem} \mathrm{Kalbe}$.

Lit.: Pasquet-Labrone, Observat. sur un cas de monstre double autositaire. L'union médic. 1875. No.128. - Gurlt, a. a. 0. S. 58.

44. Gastrodidymus, Gt. Psodymus, St. Hil.

Diese höchst selten vorkommende Art von Missgeburt habe ich jetzt nur einmal, bei einem Kalbe, untersucht; es ist die Form mit acht Extremitäten.

Lit.: Gurlt, a. a. 0. S. 58.

45. Ischiopagus, St. Hil. Hypogastrodidymus, Barkow.

Drei neue Fälle bei Menschen sind beobachtet worden. Nach einer Mittheilung von Hackedorı lebte ein Kind 3 Wochen.

Lit.: A. Hackedorn, Bicephalic monstrosity. (Ischiopagus tripus). Philade]pb. med. and surgic. reporter. 1870. No. 19. - C. Ellis, Autopsy of the double monster (Ischiopagus tripus). Boston med. and surgic. Joura. 1871. Octobr. 7. - A. Klein, Hypogastrodidymus. Wiener medic. Presse. 1871. No. 52.

46. Pygopagus, St. Hil. Pygodidymus, Gt.

Einige Beobachtungen von fast erwachsenen Zwillings-Missgeburten (Mädchen) dieser Art, und ron Thieren nur ein Fall (Kalb) sind anzuführen.

Lit.: F. Simpson, 0n the Siamese and other viable twins. Brit. med. Journ. 1869. Febr. March. - J. B. Jacks on, The Carolina sisters. Bost. med. and surgic. Journ. 1869. Jun. Jul. - Tardi e u, Mille-Christine. Bull. de l'academ. de médec. 1874. No. 2. - Joly et Peyrat, Etudes sur un monstre de genre Pygopage. Ibid. No. 3. - Gurlt, a. a. 0. S.61. Taf. XX. Fig. 116, 117.

III. Doppel-Missgeburten mit Trennung am unteren (hinteren) Ende des Körpers.

\section{Dipygus s. Monocephalus, Gt.}

Diese bei Menschen äusserst selten vorkommende Form ist bei den Thieren vielfach beobachtet, und zwar in den verschiedenen Abstufungen, die durch den mehr oder minder hohen Grad der Verdoppelung der Wirbelsäule vom Kreuzbeine anfangend und bis zum Atlas vorschreitend, jedoch so, dass der Allas nicht vollständig doppelt ist, weil das Hinterhauptsbein immer einfach ist. Es sind nur Beobachtungen an Thieren anzuführen.

Lit.: 0 tto, a. a. 0. S. 191-210. - Thiernesse, Description d'un monstre double monocéphalien. Bullet. de l'academie roy. de médec. de Belgique, 
T. VII. No. 9. - A. Schmidt, Ovis bicorporis descriptio. Dissert. Dorpati 1858. - Joly, Une lacune dans la serie teratolog. remplie. Compte rendu 1875. No. 5. - Dareste. Ibid. No. 6. - Gurlt, a, a. 0. S. 43-46.

48. Dihypogastrius, Barkow. Octopus, Gt.

Von menschlichen Missgeburten dieser Art ist keine Beobachtung aus nener Zeit mitgetheilt; desto zahlreicher sind die bei Thieren vorgekommenen Fälle.

Die erste Form, mit 2 Gesichten (Janiceps, St. Hil. Oct opus Janus, Gt.) ist weniger häufig beobachtet, aber als neu sind die Abänderungen zu betrachten, wo das eine Gesicht cyclopisch ist, oder wo beide Gesichte fehlen und nur die verschmolzenen Hirnschädel und vier Ohren vorhanden sind.

Lit.: 0tto, a. a. 0. p. 196-207. - Gurlt, a. a. 0. S. 53, 54. Taf. XVII.

Fig. 106-110. Taf. XVIII. Fig. 102-105. - Panum in diesem Archiv Bd. 72. Hft. 1. S. 69 ff. Taf. I.

Von der zweiten Form, nur ein Gesicht, aber vier Ohren (Synotus, St. Hil. O ct. quadriauritus, Gt.), ist die Zahl der Fälle schon grösser, und hier kommen vier Abänderungen vor, nämlich: mit dem vollständigen Gesicht, mit dem cyclopischen Gesicht, ohne Gesicht, ohne Unterkiefer.

Lit.: Otto, a. a. 0. p. $196-212$. Gurlt, a. a. 0. S. 55. Taf. XIX. Fig. 111-112.

Die dritte Form, mit einem Gesicht und zwei Ohren (Deradelphus, St. Hil. 0ct.bjauritus, Gt.) ist die am bäufigsten vorkommende und von ihr sind keine Abänderungen am Kopfe bekannt. Lit.: Otto, a. a. 0. S. 192-195. - Gurlt, a. a. 0. S. 56 .

\section{Craniopagus, Först. Symphyocephalus, Bark.}

E in von Badger beschriebener, hierher gehörender Fall ist anzuführen. Beide Kinder waren normal gebildet und nur durch die Haut an den Köpfen verbunden. Einen zweiten, die Menschen betreffenden Fall hat Doenitz beschrieben. Die Kinder (Fötus) sind an den Scheiteln verbunden, das eine ist normal gebildet, das andere sebr defect.

Derselbe beschreibt auch den Embryo aus einem drei Tage bebrüteten Hühnerei.

Lit.: G. Badger, Gase of monstrosity. New-York med. recorder. 1869. June 1.

- W. Doenitz, Beschreibung und Erläuterung von Doppelmissgeburten. Reichert's a.du Bois-Reymond's Archiv 1866. Taf.XII, XIV. 
IV. Doppel-Missgeburten mit Trennung am oberen und unteren Ende des Körpers.

50. Tetrascelas, Gt. Diprosopus dihypogastrius, Bk.

Es sind nur zwei neue Fälle von Thieren beobachtet, nämlich eine Lamm- und eine Schweine-Missgeburt und zwar die Form mit Doppelgesicht (Tetr. bifacialis, Gt.).

Lit. : P. L. Panum in diesem Archiv Bd. 72. S. 73. Taf. II. - Gurlt, a. a. 0. S. 57 .

51. Thoracodidymus, Gt. Sternopages, St, Hil.

Diese bei Mensehen ziemlich häufig vorkommende Art von Missgeburt ist in neuer Zeit in 6 Fällen beobachtet worden. Auch bei den Thieren sind diese Fälle nicht selten. Von diesen sind einige auffallende Abweichungen von der gewöhnlichen Form zu verzeichnen. In 2 Fällen fehlte der Kopf des rechten Körpers (bei einem Doppelkalbe und einem Doppellamme) und der Atlas dieses Körpers geht vorn in einen stumpfen, abgerundeten Fortsalz aus. Bei einem anderen Doppellamme fehlt die ganze hintere Körperhälfte vom letzten Rückenwirbel an. Diese Defecte sind wahrscheinlich durch eine intrauterine Abschnärung entstanden.

Lit.: W. Doenitz, Beschreibung und Erläuterung von Doppelmissgeburten. Re i chert's a. da bois-Reymond's Arehiv. 1865. Taf. II, HI. - H. 0. Harcy and R. H. Fitz, A case of double monstrosity. Boston med. and surgic. Journ. 1871. Jul. 13. - B. Thomps on, An other monstrosity. Philadelph. med. and surgic. reporter. 1871. Jan. 7. - E. Kortüm, Beschreibung einer Doppelmissgeburt. Dieses Archiv Bd.62. S. 441. - Böttcher, Ein Fall von Doppelmissbildung. Dorpat. medic. Zeitschr. Bd.V. Hft. 4. 1875. - Lürmann, Ein Fall von Doppelmissbildong. Dissert. Kiel 1874. - Gurlt, a. a. 0. S.59, 60. Taf. XIX. Fig. 113-11 j.

\section{Xiphopages, St. Hil. Thoracopagus xiphopagus, Först.}

Diese nur bei Menschen beobachtete Missbildung ist in neuer Zeit fünfmal vorgekommen.

Lit.: A. Böttcher, Zur Anatomie der Xiphopagen-Doppelbildungen. Dorpat. med. Zeitschr. 1871. II. S. 105. - Engels, Ueber Xiphopagen. Dissert. Berlin 1874. - Elwood, A remarcable double monstrosity. Philadelph. med. reporter. 1874. - Harley, United twin monstrosity. The brit. med. Journ. 1875. Apr. 17. - Montgomery, A case of double monster. Philadelph. med. Times. 1875. Mai 1. 
V. Monstra per implantationem duplicia. Monstra parasitica.

53. Heterodidymus, Gt. Heteradelphus, St. Hilaire.

Eine neuere Beobachtung über menschliche Missgeburtèn dieser Art ist nicht $z \mathfrak{u}$ verzeichnen. Von thierischen Missgeburten habe ich vier Beobachtungen gemacht, bei allen fehlte dem Pasiten der Kopf, er hatte aber vier Beine. Otto, a. a. 0. S. 251-258 hat mehrere Fälle von Thieren beschrieben.

Lit.: Gurlt, a. a. 0. S. 46. Taf. XVIII. Fig. 95, 96.

54. Cryptodidymus, Gt. Foetus in foetu.

Es ist mir hiervon nur e in Fall vorgekommen, in welchem sich bei einem 8 Tage alten weiblichen $\mathrm{Kalbe}$ das linke Hinterbein mit einem Beckenrudiment in der Bauchhöhle vorfand. Von Eingeweiden war nur ein unvollständiger Uterus vorhanden und mit dem Beine verbunden.

Lit.: Gurlt, a. a. 0. S. 61. Taf. XX. Fig. 118, 119.

55. Cephalomeles, St. Hil. Omphalo-Cranodidymus, Gt.

Bei einem erwachsenen Schafe jst ein sebr verkümmertes Bein, ob Vorder- oder Hinterbein ist nicht zu bestimmen, durch einen Gefässstrang, der aus einer Oeffnung am Scheitel hervortritt, mit der Dura mater des kleinen Gehirns verbunden. Bei einer Ente ist das Bein auf dem Kopfe ziemlich vollständig.

Lit.: Gurlt, a. a. 0. S. 60 .

56. Epignathus, auct. Heterocephalus epignathus, Gt.

Hess hat zwei hierher gehörende Fälle beschrieben. Bei einem Kinde enthielt die aus dem Munde hervorgetretene Geschwulst nur Rudimente von Knochen. Bei dem zweiten $K$ inde waren unvollständige Extremitäten und Eingeweide vorhanden.

Ich habe ein Kalb untersucht, bei welchem an das linke Flügelbein ein unvollkommener Oberkiefer mit Backenzähnen angewachsen ist.

Lit.: W. Hess, Beitrag zur Casuistik der Geschwälste mit zengungsähnlichem Inbalt. Dissert. Giessen (ohne Jahreszahl). - Gurlt, a. a. 0. S. 41. Taf. XVI. Fig. 93, 94. 


\section{Monstra triplicia.}

57. Melotridymus, Gt.

Den einzigen Fall berichtet Ja gor. Ein Kind mit regelmässigem Oberkörper hatte 6 Beine und lebte 5 Tage.

Lit.: Jagor, Pluralitat. monstros. Dieses Archiv Bd. 50. S. 296.

Zur Literatur über menschliche and thierische Missbildungen ist noch anzuführen:

Descriptive Catalogue of the Teratological Series in the Museum of the Royal College of Surgeons of England. By B. Thompson Lowne. London 1872.

\section{XXV.}

\section{Kritische Bemerkungen zur Aetiologie der Area Celsi.}

Von Dr. Hans Buchner in München.

Unter Area Celsi (auch Alopecia areata etc.) verstehe ich jenes Leiden der behaarten Haut, bei welchem an mehr oder minder deutlich kreisförmigen Stellen, die allmählich sich vergrössern, ein Lockerwerden und Ausfallen der Haare eintritt, ohne dass die Haut selbst auffälligere Veränderungen erkennen liesse ${ }^{1}$ ).

Anknüpfend an die von Pincus ${ }^{2}$ ) und neuerdings von Michelson ${ }^{3}$ ) gegebenen Zusammenstellungen unserer Kenntnisse über diesen Prozess wird im Folgenden gezeigt werden, dass der jetzt herrschenden ätiologischen Beurtheilung der Area Celsi gewichtigere Bedenken entgegenstehen, als solche jemals gegen die ältere parasitäre Theorie derselben vorgebracht werden konnten.

1) Ich betone besonders das allmähliche Umsichgreifen des Prozesses, da dies, wie wir sehen werden, die wesentlichste Eigenthümlichleit des Leidens darstellt, und doch von den meisten neueren Autoren als etwas Nebensächliches betrachtet wurde. Ausgesehlossen von der Bezeichnung als Area Celsi halte ich alle jene Formen des Haarschwunds, auf welche die obigen Kennzeichen nicht anwendbar sind, insbesondere jene, bei denen der progrediente Charakter nicht zu erweisen ist.

2) Deutsche Klinik 1869. S. 1, 15, 126, 141, 163.

3) Sammlung klin. Vorträge von R. Volkmann. No. 120. 1877. 